S. de Ribaupierre

K. Meagher-Villemure

J. G. Villemure

J. Cotting

P. Y. Jeannet

F. Porchet

E. Roulet

J. Bloch

\title{
The role of posterior fossa decompression in acute cerebellitis
}

Received: 3 May 2004

Published online: 1 June 2005

(C) Springer-Verlag 2005

S. de Ribaupierre $(\bowtie) \cdot J$. G. Villemure ·

F. Porchet ·J. Bloch

Département de Neurochirurgie,

CHUV, Rue du Bugnon,

1011 Lausanne, Switzerland

e-mail: sderib@hotmail.com

Tel.: +41-21-3142626

Fax: +41-21-3142595

K. Meagher-Villemure

Pathology Department, CHUV,

Lausanne, Switzerland

\author{
J. Cotting \\ Pediatric Intensive Care Unit, CHUV, \\ Lausanne, Switzerland \\ P. Y. Jeannet - E. Roulet \\ Pediatric Neurology Department, \\ CHUV, \\ Lausanne, Switzerland
}

\begin{abstract}
Background: We present two cases of children who were diagnosed with cerebellitis with acute cerebellar swelling. This rare pathology is potentially fatal, and no clear treatment guidelines are described in
\end{abstract}

the literature. Discussion: Considering our experience, we discuss the different therapeutic strategies and propose aggressive surgical measures consisting of external ventricular drainage and posterior fossa decompression in case of failure of early response to medical treatment to limit secondary cerebellar and brainstem lesions.
Keywords Cerebellitis · CNS infection $\cdot$ Posterior fossa decompression $\cdot$ Hydrocephalus

\section{Introduction}

Para-infectious involvement of the central nervous system is rare, but para-infectious cerebellitis with acute cerebellar swelling is exceptional and deserves special attention because the treatment modalities can diverge from medical care to aggressive surgical decompression. We report here two cases of children who presented with acute cerebellitis and brainstem involvement, and whose outcome was good with ventricular drainage and posterior fossa decompression. Treatment options are discussed in this potentially fatal pathology.

\section{Case reports}

\section{Case 1}

A 6 1/2-year-old girl, with known migraine disorder, presented with a 2-day history of pulsating headache, photophobia, nausea and onset of vomiting that morning. Her neurological and fundoscopic exams were normal, and she was admitted for observation with a diagnosis of severe migraine. On day 2, her headache increased despite pain medication, and she developed meningeal signs, upper limb dysmetria, and a temperature of $37.8^{\circ} \mathrm{C}$. A cerebral CT scan showed posterior fossa meningeal enhancement and diffuse cerebellar swelling, with a slight upward trans-tentorial and downward trans-foramen magnum herniation. Cisternae were still visible, and there was no hydrocephalus (Fig. 1a). The child was admitted to the intensive care unit with a diagnosis of cerebellitis with acute cerebellar swelling, and dexamethasone treatment $(0.5 \mathrm{mg} / \mathrm{kg} \mathrm{q} 6)$ was started. On admission, white blood count was 6,000 . By day 3, cerebral MRI confirmed the diffuse cerebellar swelling, with T2-hyperintense lesions of both cerebellar hemispheres predominantly on the right side (Fig. 2a, b), without venous or arterial abnormalities. She worsened progressively during the fourth day with a decreased level of consciousness. Ocular bobbing and bradycardia were observed, and brainstem involvement was suspected. A repeated cerebral CT scan showed increased cerebellar swelling with disappearance of basal cisterns, a deformed fourth ventricle and moderate tri-ventricular hydrocephalus without trans-ependymal resorption (Fig. 1b). An external ventricular drainage was inserted in the right frontal 
Fig. 1 Case 1: axial cerebral CT scan, through the lateral and the third ventricles showing a day 1 , normal ventricular size; b day 3 , enlargement of the ventricular system; c day 4, increased hydrocephalus despite external ventricular drain (in place and functioning)
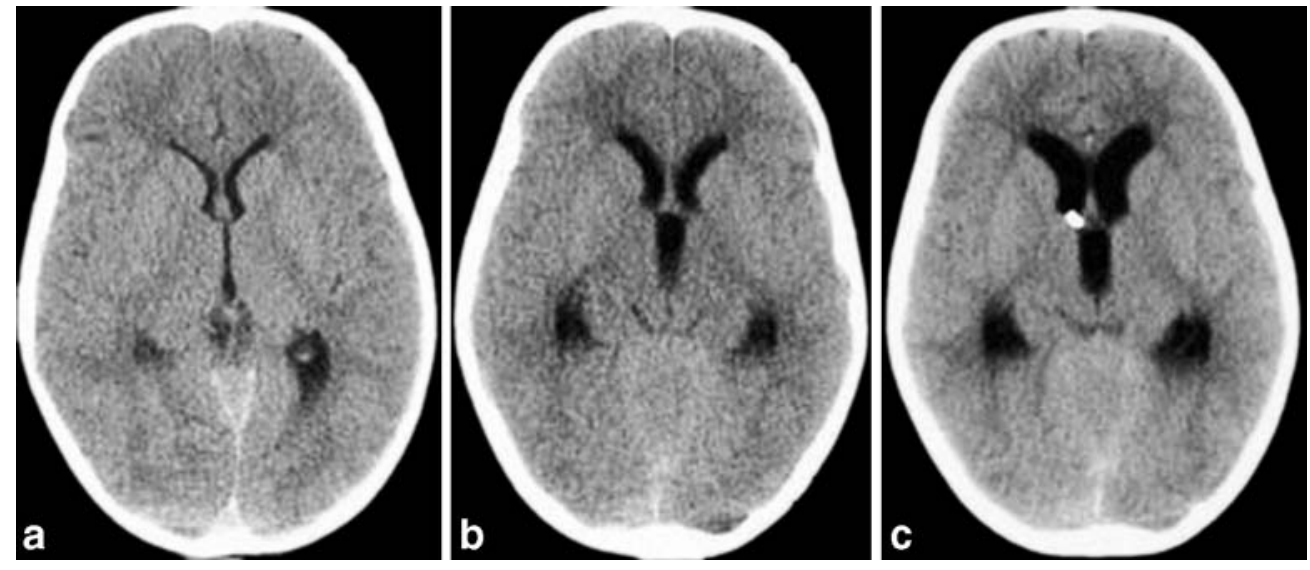

horn with an opening pressure of $15 \mathrm{~cm} \mathrm{H}_{2} \mathrm{O}$. The drain was collecting around $2 \mathrm{ml} / \mathrm{h}$ at $+15 \mathrm{~cm}$. CSF showed less than one cell, with $35 \mathrm{mg} / \mathrm{l}$ for proteins and $7 \mathrm{mmol} / \mathrm{l}$ for glucose. White blood count increased to 18,000 , and CRP was $58 \mathrm{mg} / \mathrm{l}$. On day 5 , her clinical exam had not improved, and a repeated cerebral CT scan showed an enlargement of the ventricles despite good positioning and functioning of the drain (Fig. 1c). Since posterior fossa was very full with herniation and brainstem compression, a posterior fossa decompression with resection of the cerebellar tonsils was then performed to avoid secondary brainstem lesions. Material from surgical biopsies consisted of fragments of cerebellar cortex with granular cell layer. There was a mixed chronic inflammatory infiltrate focally in the arachnoid and few perivascular cuffing of

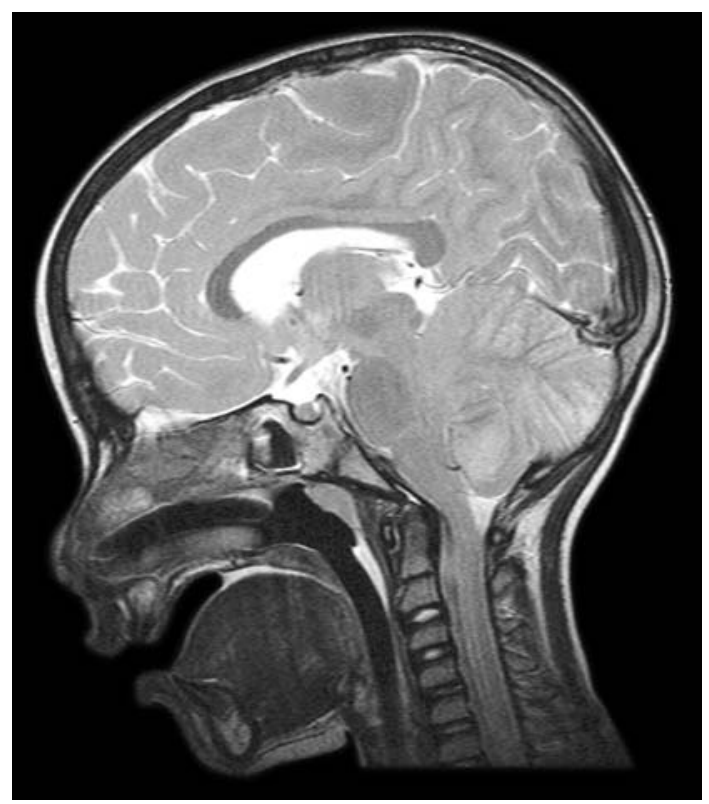

Fig. 2 Case 1: sagittal $\mathrm{T}_{2}$-weighted MRI (day 1) showing fullness of the posterior fossa with upward trans-tentorial and downward transforamen magnum herniation the same type of infiltrate in the white matter. An immunohistochemistry study of the type of inflammatory infiltrate was positive for $\mathrm{T}$ lymphocytes (CD3), B lypmhocytes (CD20) and numerous macrophages (CD68) (Fig. 3). The biopsy PCR [Lyme, Epstein-Barr virus (EBV) and varicella] remained negative. Different etiologies were searched, but Lyme and EBV serologies were negative, and IgG for varicella were detected but compatible with the primoinfection at the age of three.

By day 6 the patient clinically started to improve and was extubated, and dexamethasone was decreased progressively. Cerebral MRI showed a slight decrease in ventricular size. Cerebellar lesions were demarcated, and pinpoint bulbar lesions appeared, which confirmed the suspicion of brainstem involvement (Fig. 4). CRP and leukocytes decreased as well. The external ventricular drainage was withdrawn on day 13 . The child started rehabilitation with daily progress, but had persistence of ataxia and hypopho-

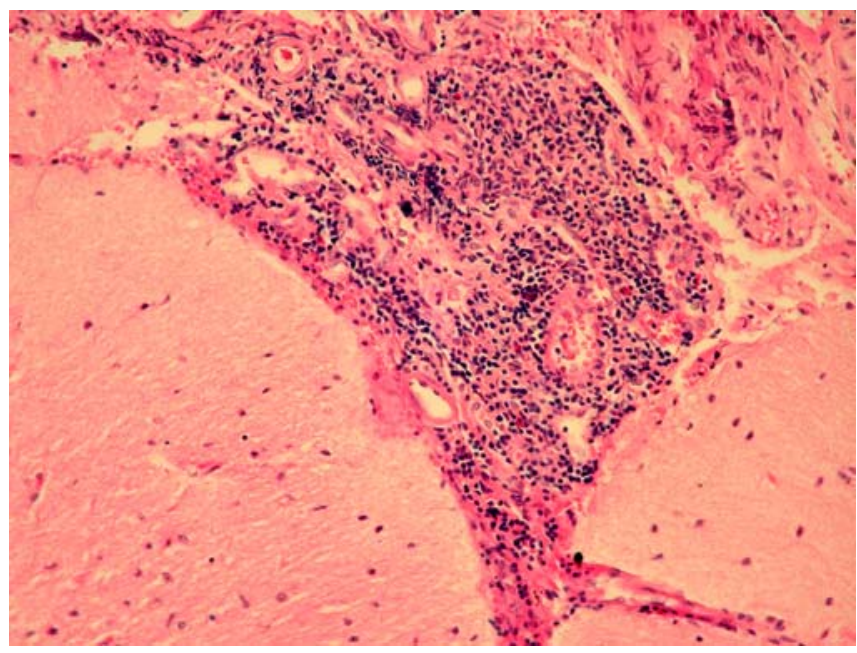

Fig. 3 Case 1: chronic inflammatory infiltrate in the arachnoid over the cerebellar cortex. HE $\times 100$. Cerebellar granular layer and white matter with perivascular cuffing by lymphocytes and plasma cells. $\mathrm{HE} \times 100$ 


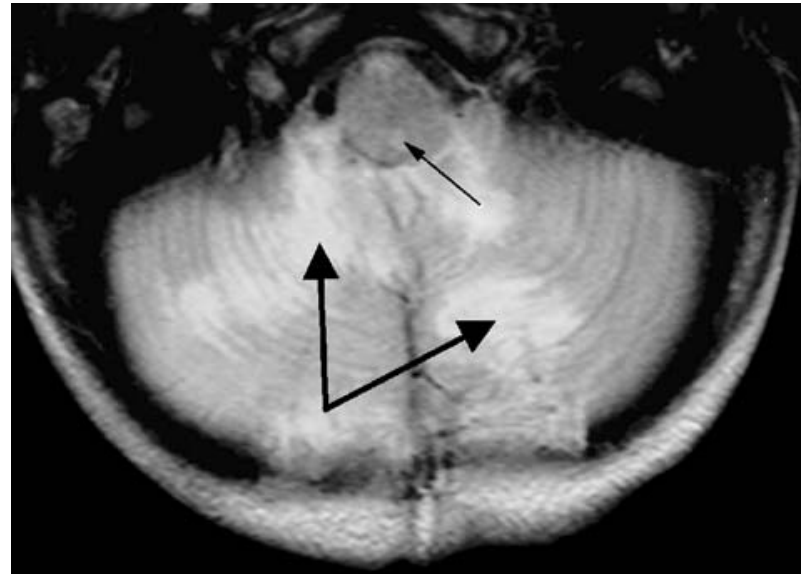

Fig. 4 Case 1: axial $\mathrm{T}_{2}$-weighted MRI (day 1) showing hyperintensities in the cerebellar hemispheres (large arrows), and involvement of the bulbar region (thin arrow)

nia. Before being discharged home (day 28), a repeated cerebral CT scan showed a slight decrease in ventricular size, but a large subdural or subarachnoid posterior fossa collection without mass effect, which was interpreted as secondary to cerebellar atrophy (Fig. 5). Nineteen months later, the child showed no neurological deficits, and her voice was back to normal.

\section{Case 2}

A previously healthy 14-year-old girl, with a childhood history of varicella, mumps and measles without any com-

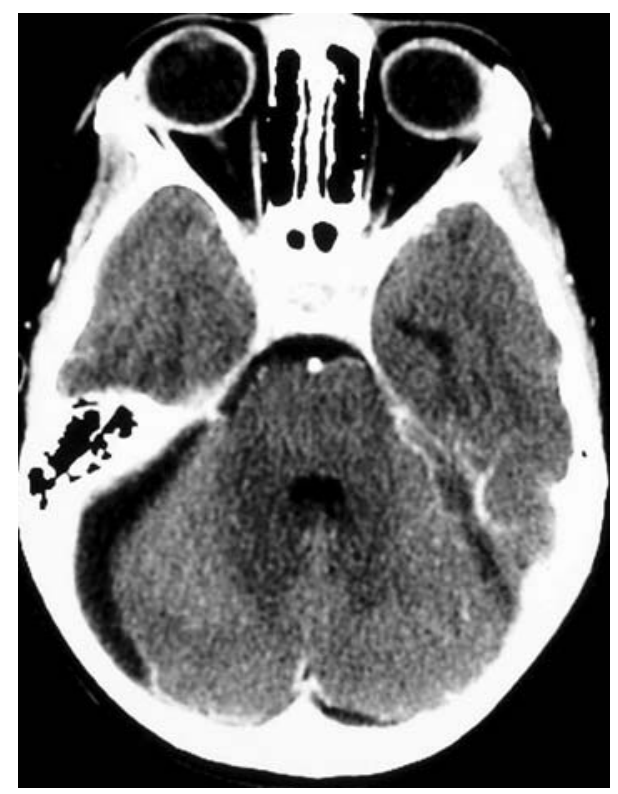

Fig. 5 Case 1: enhanced axial cerebral CT scan (1 month later) showing cerebellar atrophy plications, and immediate past history of aphthous ulcers followed by sinusitis treated with Augmentin (amoxicillin and clavulanate potassium) and Klacid (Clarithromycin), was admitted to the hospital with acute pulsating headaches, photophobia and nausea. The family had noticed a change in her voice in the last few days. On physical exam, she was afebrile with a heart rate down to 35 beats $/ \mathrm{min}$; her neurological and fundoscopic exams were normal. The cerebral CT scan showed fullness of the posterior fossa and a slight upward trans-tentorial and downward trans-foramen magnum herniation, as well as hydrocephalus. MRI showed bilateral cerebellar hemisphere hyperintensities on fluidattenuated conversion recovery (FLAIR) sequences (Fig. 6). White blood count was 9,000 , and CRP was $13 \mathrm{mg} / \mathrm{l}$. She was diagnosed with acute cerebellitis and received a treatment of dexamethasone $(0.5 \mathrm{mg} / \mathrm{kg})$ every $4 \mathrm{~h}$. On day 1 her clinical status worsened, and she underwent placement of an external ventricular drainage, with an opening pressure lower than $15 \mathrm{~cm} \mathrm{H}_{2} \mathrm{O}$, and posterior fossa decompression, with resection of the cerebellar tonsils and cerebellar biopsies. The sample collected from the surgery showed foci of inflammatory reaction at every level from the arachnoid to the deep white matter as well as significant microglial cells activation within the cerebellar cortex. Immunohistochemistry results confirmed the mixed type of inflammatory infiltrate and negativity for herpes virus, cytomegalovirus, and EBV by in situ hybridization. On ultrastructure study, no viral particle could be identified (Fig. 7). The serologies (Lyme, EBV and varicella) remained negative. The maximum white blood count during hospitalization was 13,000 and CRP was $14 \mathrm{mg} / \mathrm{l}$.

On day 2 the patient gradually improved clinically. A repeated cerebral CT scan showed no decrease in the hy-

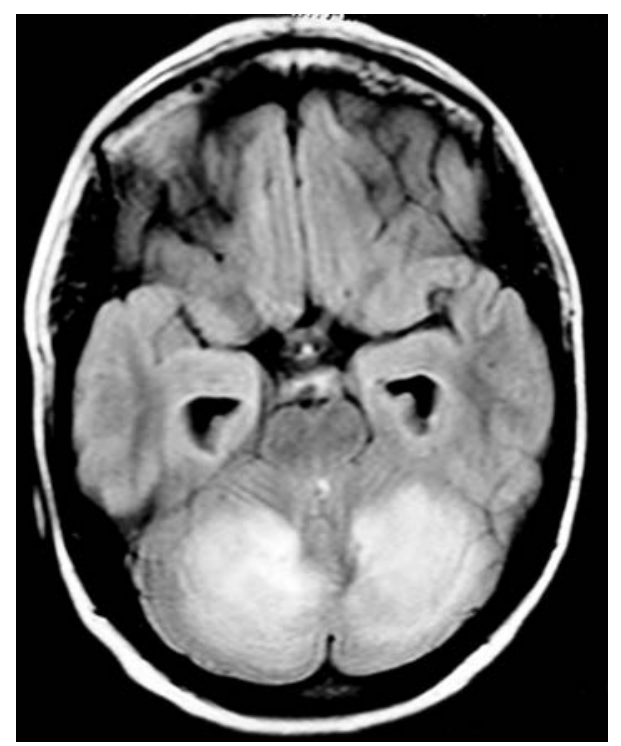

Fig. 6 Case 2: axial T2-FLAIR MRI (day 1) showing hypersignals in both cerebellar hemispheres 


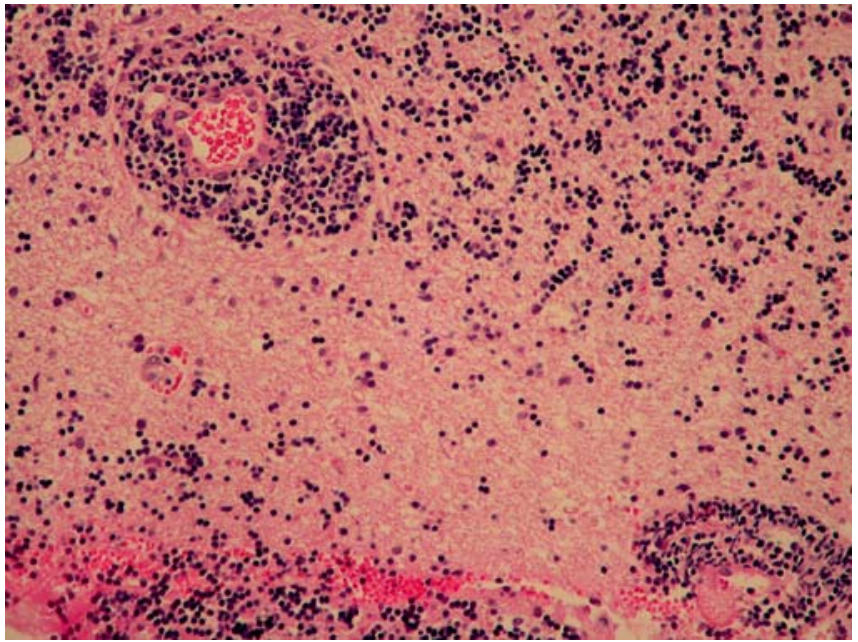

Fig. 7 Case 2: numerous microglial cells in the cerebellar cortex. Perivascular cuffing of lymphocytes and plasma cells at the junction of the granular cells layer and the white matter. HE $\times 200$

drocephalus despite good positioning (confirmed by the CT scan) and functioning of the drain $(5-10 \mathrm{ml} / \mathrm{h})$.

By day 9 the cerebral CT scan showed a slight decrease in the ventricular size, with a posterior fossa subdural collection having the same density as CSF. She continued to improve clinically with a rapidly decreasing ataxia and had no complications. The clinical follow-up at 13 months revealed a normal neurological exam, and the patient was headache-free.

\section{Discussion}

Cerebellitis with acute cerebellar swelling in children has rarely been reported in the literature. In all of the cases we have reviewed, the pathology seemed to be limited to the cerebellum. The involvement of the brainstem observed in our first case is exceptional and underlines the severity of the process without necessarily being a factor of poor prognosis. Typically, there are bilateral hemispheric cerebellar $\mathrm{T}_{2}$-weighted hyperintensities and cerebellar swelling on MRI, with or without gadolinium enhancement $[1,2]$.

The etiology is thought to be para-infectious (after varicella, EBV or enteric fever [3-7]), but in most cases no specific agent is found, as observed in our patients.

Considering the management of this condition, there are no established rules, and we believe that every case should be considered individually. Therapeutic options are either medical with high dose steroids and close monitoring, or surgical with either external ventricular drainage, posterior fossa decompression, or both. Medical management is probably sufficient in milder cases of non-progressive cerebellitis with no clinical or radiological progression $[8,9]$.
However, if the disease is severe with hydrocephalus and/ or threat of brainstem compression, this approach is not sufficient, and surgical options have to be considered.

Most of the authors consider hydrocephalus associated with cerebellar swelling as purely obstructive at the level of the fourth ventricle. This is probably the case when the opening ventricular pressure is high, and drainage may be the adequate treatment $[4,10]$. However, in the presence of obstructive hydrocephalus, there is a risk of upward herniation with ventricular drainage if the drain is too low or if the patient is not carefully monitored. There are cases of unexpected low opening pressure, as presented here, and as reported by Asenbauer et al. [11]. In the latter, hydrocephalus may result from a combination of CSF flow obstruction and mal-resorption. Hydrocephalus, in such cases, is possibly associated with a gradual inflammatory subarachnoid space obstruction which leads to a progressive ventricular size enlargement with regulation of the intracranial pressure, as observed in the adult normotensive hydrocephalus, even though the situation is relatively acute. The absence of trans-ependymal resorption seen in our patient, as well as in the two cases reported by Roulet Perez et al. [12], is another argument in favor of this theory. Moreover, ventricular size enlargement, despite posterior fossa decompression, as seen in our patients, reinforces our hypothesis.

External ventricular drainage associated with corticosteroids can be efficient; unfortunately, a few patients do not respond to this treatment. There are reported cases of fatal issues despite efficient drainage [12, 13]. In the latter, neurological deterioration is likely secondary to direct brainstem compression from the cerebellar swelling. Posterior fossa decompression therefore appears to be indicated in some cases of acute cerebellitis.

In the presence of fourth ventricle obliteration, hydrocephalus, and low intracranial pressure, posterior fossa decompression should be considered an emergency if the child does not immediately improve after CSF drainage. This approach was only reported once previously [11] and its aim is to avoid brainstem lesion secondary to posterior fossa mass effect. The timing of surgical decompression is crucial, and surgery should be performed early to avoid brainstem compression and irreversible damage. In both of our cases, this approach was therapeutic with good clinical postoperative outcome.

After the acute phase and adequate treatment, recovery is generally good; however, it is difficult to predict which patient is going to present cerebellar sequellae $[1,14]$. There must be a correlation between the severity of the process and the long-term evolution; nevertheless, we think that early posterior fossa decompression may not only be lifesaving, but may also reduce the occurrence of secondary lesions and thus improve the clinical outcome. 


\section{Conclusion}

Cerebellitis with acute cerebellar swelling is a rare pathology that deserves special attention since early recognition and adequate treatment may change the course of the disease. Progressive cerebellar swelling may lead to hydrocephalus and/or brainstem compression.

In both cases presented here, surgical management was followed by favorable outcome. We are not able to for- mulate therapeutic guidelines on two isolated cases; however, after this experience, we believe that prompt surgical management should be considered in acute cerebellitis with failure of medical treatment. In the present cases, external ventricular drainage was not sufficient, and clinical worsening led us to proceed to posterior fossa decompression followed by rapid neurological improvement.

\section{References}

1. Bakshi R, Bates VE, Kinkel PR, Mechtler LL, Kinkel WR (1998) Magnetic resonance imaging findings in acute cerebellitis. Clin Imaging 22:79-85

2. Montenegro MA, Santos SL, Li LM, Cendes F (2002) Neuroimaging of acute cerebellitis. J Neuroimaging 12(1): 72-74

3. de Fraiture DMI, Sie TH, Boezeman EHJF, Haanen HCM (1997) Cerebellitis as a uncommon complication of infectious mononucleosis. Neth $\mathrm{J}$ Med 51:79-82

4. Hamada H, Kurimoto M, Masuoka T, Hirashima Y, Endo S, Harada J (2001) A case of surgically treated acute cerebellitis with hydrocephalus. Childs Nerv Syst 17:500-502
5. Drost G, Verrips A, Thijssen HO, Gabreels (2000) Cerebellar involvement as a rare complication of pneumococcal meningitis. Neuropediatrics 31(2): 97-99

6. Majda-Stanislawska E (2000) Mumps cerebellitis. Eur Neurol 43(2):117

7. Mario-Ubaldo M (1995) Cerebellitis associated with Lyme disease. Lancet 345(8956): 1060

8. Aylett SE, O'Neill KS, De Sousa C, Britton J (1998) Cerebellitis presenting as acute hydrocephalus. Childs Nerv Syst 14:139-141

9. Gohlich-Ratmann G, Wallot M, Baethmann M, Schaper J, Roggendorf M, Roll C et al (1998) Acute cerebellitis with near-fatal cerebellar swelling and benign outcome under conservative treatment with high dose steroids. Eur J Paediatr Neurol 2(3):157-162

10. Horowitz MB, Pang D, Hirsch W (1991) Acute cerebellitis: case report and review. Pediatr Neurosurg 17(3):142-145
11. Asenbauer B, McConachie NS, Allcutt D, Farrell MA, King MD (1997) Acute near-fatal parainfectious cerebellar swelling with favourable outcome. Neuropediatrics 28(2):122-125

12. Roulet Perez E, Maeder P, Cotting J, Eskenazy-Cottier AC, Deonna T (1993) Acute fatal parainfectious cerebellar swelling in two children. A rare or an overlooked situation? Neuropediatrics 24(6):346-351

13. Levy EI, Harris AE, Omalu BI, Hamilton RL, Branstetter BF, Pollack IF (2001) Sudden death from fulminant acute cerebellitis. Pediatr Neurosurg 35 (1):24-28

14. Hayakawa H, Katoh T (1995) Severe cerebellar atrophy following acute cerebellitis. Pediatr Neurol 12:159-161 